\title{
Adhesive Impact of Micromechanical Surface Contact
}

\author{
Biswajit Bera \\ Department of Mechanical Engineering National Institute of Technology Durgapur, India
}

\begin{abstract}
In this study, a new generalized adhesion theory of solid sphere is developed to investigate adhesive impact of head-disk surfaces. On the basis of this adhesion theory, deformation and restoration work done is evaluated for multiasperity adhesive impact of head-disk surfaces. From the plot of coefficient of restitution with mean separation, its found COR decreases gradually, with decrement of mean separation approaching to zero value which represents unique characteristic for head-disk impact. It indicates there is possibility of seizure of head-disk due to high adhesion at the interface for high impact.
\end{abstract}

Keywords: Adhesion theory, Deformation work done, Restoration work done, Coefficient of restitution

\section{INTRODUCTION}

In hard disk drives, one of the main technological challenges is the stability and reliability of head disk interface (HDI), where the recording head and slider move in close proximity to the magnetic recording media at ultrahigh speed rate. The read/write element is situated at the trailing edge of the slider. The magnetic layer of the disk where the data are stored is overcoated with a diamondlike-carbon (DLC) for wear protection. The thin overcoat is usually covered by 1-2 $\mathrm{nm}$ of lubricant layer to reduce the friction and wear between the carbon overcoat and the recording head. In the disk drive, the slider flies a few nanometers over the disk surface, which is designed to maintain stability in the flying height as the head moves over the disk surface. In order to achieve higher density recording of $100 \mathrm{Gbit} / \mathrm{in} 2$ and possibly $1 \mathrm{Tbit} / \mathrm{in} 2$; the distance between the flying slider and the disk surface has to reduce further, down to flying-heights of approximately $10 \mathrm{~nm}$ and $6.5 \mathrm{~nm}$, respectively, to allow maximum magnetic storage capacity. However, reducing the flying height of the recording head to achieve higher recording densities and storage capacities may cause the intermittent contact of operation. Generally, impacts occur at the corners of the recording head with high rebound velocity. Such impact could result in high contact forces which can cause permanent mechanical disk damage and magnetic erasures.

Generally, considering rounded corner of head, head - disk impact theory are available in literature [1]. But impact theory of head-disk considering rough surface are rarely available. First, Chang and Ling [2] have presented an elastic-plastic model for normal impact of rough surfaces. Thereafter, Sahoo and Roy Chowdhury [3] have developed impact theory of rough surfaces in presence of adhesion considering loading and unloading. In the present study, a generalized adhesion theory of elastic sphere is developed and this is implemented in adhesive head-disk rough surface impact. Here, interfacial adhesion is considered significant way of energy dissipation.

\section{THEORETICAL FORMULATION}

2.1 New generalized adhesion theory

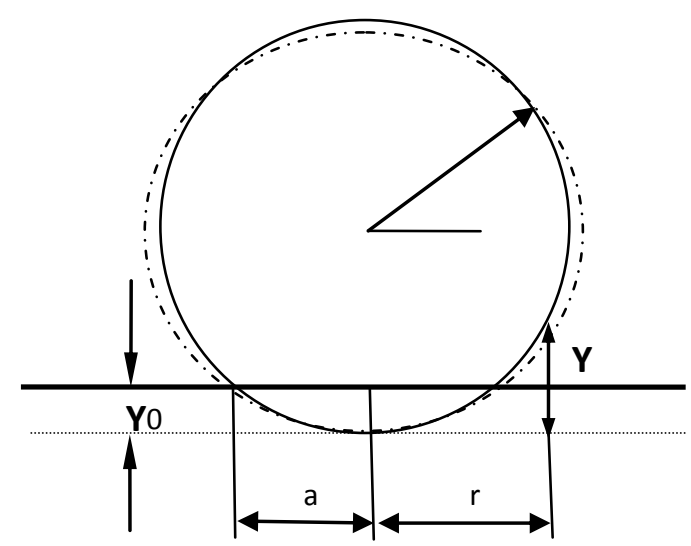

Fig.1a Contacting single asperity

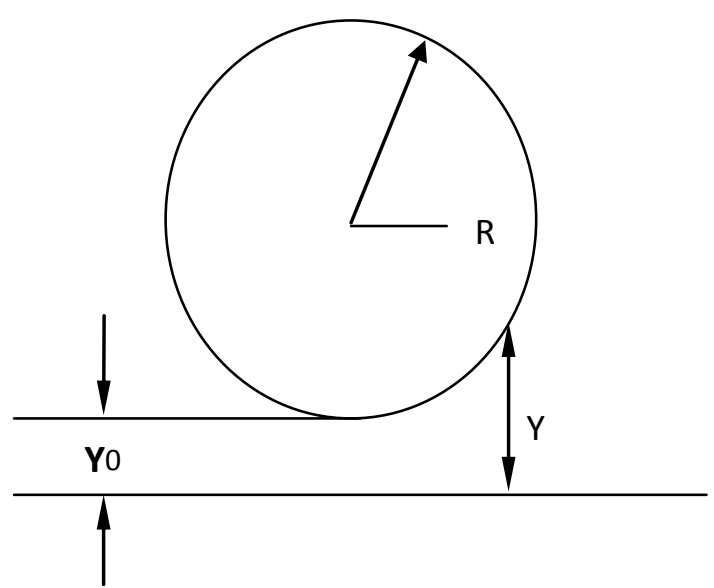

Fig.1b Noncontacting single asperity 
Spherical asperity contact with surface could be understood from adhesive contact of sphere with flat surface as shown in Fig.1a. According to DMT adhesion theory [4], when a smooth spherical solid is pressed on flat surface, it will deform followed by Hertzian theory but adhesive force will act only out side of the contact zone. According to Muller et. all. study [5], Lennad-Jones pressure profile follow the below equation.

$$
p(Y)=\frac{8}{3} \frac{\gamma}{\varepsilon}\left[\left(\frac{\varepsilon}{Y}\right)^{3}-\left(\frac{\varepsilon}{Y}\right)^{9}\right]
$$

where separation distance between sphere and flat surface, $Y=Y_{0}+\frac{r^{2}}{2 R}$ and $\varepsilon$ is the equilibrium intermolecular distance.

Now, if Hertzian deformed sphere is equivalently, considered as truncated sphere and adhesion force acts through out the surface profile, adhesive force could be calculated through integration of attractive Lennard-Jones pressure profile. Now, the adhesion force of deformed sphere is summation of adhesion force in Hertzian contact zone and adhesive force out side of the contact zone,

$$
\begin{aligned}
\text { so, } & \mathrm{F}_{\mathrm{adh}}=2 \pi \int_{0}^{\mathrm{a}} \mathrm{p}(\mathrm{Y}) \mathrm{rdr}+2 \pi \int_{\mathrm{a}}^{\infty} \mathrm{p}(\mathrm{Y}) \mathrm{rdr} \\
= & 2 \pi \int_{0}^{\infty} \mathrm{p}(\mathrm{Y}) \mathrm{rdr} \\
= & 2 \pi \int_{Y_{0}}^{\infty} p(Y) R d Y \\
= & \frac{8}{3} \pi \mathrm{R} \gamma\left[\left(\frac{\varepsilon}{\mathrm{Y}_{0}}\right)^{2}-0.25\left(\frac{\varepsilon}{\mathrm{Y}_{0}}\right)^{8}\right]
\end{aligned}
$$

The above adhesion force equation is also valid for noncontacting single asperity (Fig.1b) as Hertzian deformed profile is considered approximately spherical circle which was first mentioned by Muller et. all. [5]. The adhesion theory is generalized because adhesion is considered within and outside of Hertzian contact zone and it is applicable for both deformed and nondeformed sphere.

\subsection{Work done during impact}

First of all, Greenwood and Williamson [6] developed statistical multyasperity contact model of rough surface under very low loading condition. It is extended and based on following assumption;

i. The rough surface is isotropic.

ii. Asperities are spherical near their summits.

iii. All asperity summits have the same radius $\mathrm{R}$ but their heights very randomly.

iv. Asperities are far apart and there is no interaction between them.

v. Contacting asperities are deformed elastically and formed adhesive bond according to new adhesion theory

vi. There is no bulk deformation. Only, the asperities deform during contact.

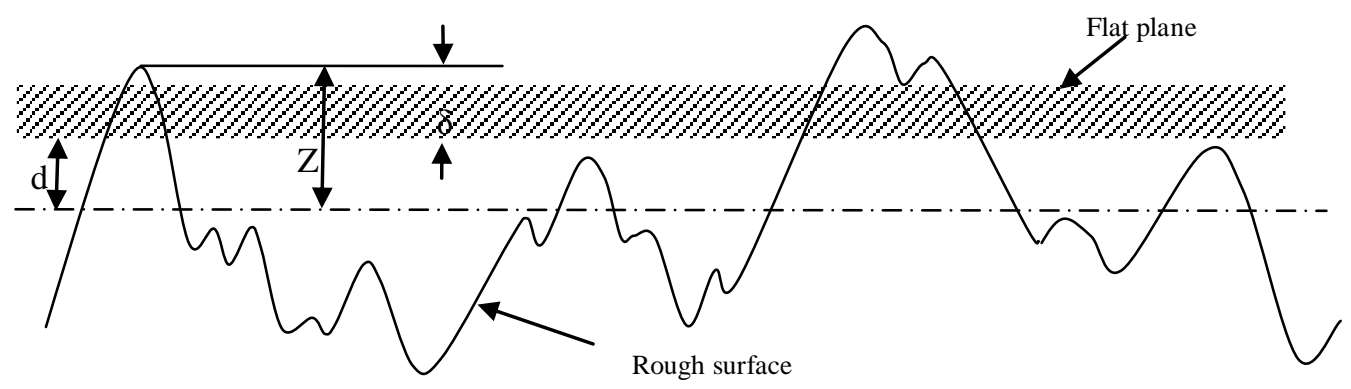

Fig.2 Rough surface contact

Multiasperity contact of rough has shown in Fig.2. According to, GW model, two rough surface contact could be considered equivalently, contact between rough surface and smooth rigid surface. Let $\mathrm{z}$ and $\mathrm{d}$ represents the asperity height and separation of the surfaces respectively, measured from the reference plane defined by the mean of the asperity height. $\delta$ denotes deformation of asperity by flat surface. Number of asperity contact is 
$N_{c}=N \int_{d}^{\infty} \phi(z) d z$

Where $N$ is total number of asperity and $\phi(z)$ is the asperity height distribution function.

\subsubsection{Deformation work done}

The elastic potential energy (strain energy) stored in a contacting asperity during deformation period is

$$
\begin{aligned}
w_{d 1}(\delta) & =\int_{0}^{\delta} F_{d 1}(\delta) d \delta \\
& =\int_{0}^{\delta}\left(K R^{0.5} \delta^{1.5}+\frac{8}{3} \pi \gamma R\left(\left(\frac{\varepsilon}{\varepsilon+\delta}\right)^{2}-0.25\left(\frac{\varepsilon}{\varepsilon+\delta}\right)^{8}\right)\right) d \delta \\
& =0.4 \mathrm{NKR}^{0.5} \delta^{2.5}+\frac{8}{3} \pi \mathrm{N} \gamma \mathrm{R} \varepsilon\left(-\left(\frac{\varepsilon}{\varepsilon+\delta}\right)+0.036\left(\frac{\varepsilon}{\varepsilon+\delta}\right)^{7}+0.964\right)
\end{aligned}
$$

Combining equation (1) and (2), the total elastic potential energy (strain energy) stored in all the contacting asperities can be obtained as

$$
\begin{aligned}
\mathrm{W}_{\mathrm{dl}} & =\mathrm{N} \int_{\mathrm{d}}^{\infty} \mathrm{w}_{\mathrm{dl}}(\delta) \phi(\mathrm{z}) \mathrm{dz} \\
& =\mathrm{N} \int_{\mathrm{d}}^{\infty}\left(0.4 \mathrm{NKR} R^{0.5} \delta^{2.5}+\frac{8}{3} \pi \mathrm{N} \gamma \mathrm{R} \varepsilon\left(-\left(\frac{\varepsilon}{\varepsilon+\delta}\right)+0.036\left(\frac{\varepsilon}{\varepsilon+\delta}\right)^{7}+0.964\right)\right) \phi(\mathrm{z}) \mathrm{dz}
\end{aligned}
$$

Dividing both side by $\mathrm{A}_{\mathrm{n}} \mathrm{K} \sigma$

$$
\begin{aligned}
\mathrm{W}_{\mathrm{d} 1}^{*} & =\int_{0}^{\infty}\left(0.4(\eta \mathrm{R} \sigma)\left(\frac{\mathrm{R}}{\sigma}\right)^{-0.5} \Delta^{2.5}+\frac{8}{3} \pi(\eta \mathrm{R} \sigma)\left(\frac{\gamma}{\mathrm{K} \sigma}\right) \varepsilon_{0}\left(-\left(\frac{\varepsilon_{0}}{\varepsilon_{0}+\Delta}\right)+0.036\left(\frac{\varepsilon_{0}}{\varepsilon_{0}+\Delta}\right)^{7}+0.964\right)\right) \phi(\mathrm{z}) \mathrm{d} \Delta \\
& =\int_{0}^{\infty}\left(0.4 \mathrm{~A}_{0} \mathrm{R}_{0}^{-0.5} \Delta^{2.5}+\frac{8}{3} \pi \mathrm{A}_{0} \mathrm{~B}_{0} \varepsilon_{0}\left(-\left(\frac{\varepsilon_{0}}{\varepsilon_{0}+\Delta}\right)+0.036\left(\frac{\varepsilon_{0}}{\varepsilon_{0}+\Delta}\right)^{7}+0.964\right)\right) \frac{1}{\sqrt{2 \pi}} \exp \left[-\frac{(\mathrm{h}+\Delta)^{2}}{2}\right] \mathrm{d} \Delta
\end{aligned}
$$

The Lennard Jones potential energy is stored in a noncontacting asperity during deformation period is

$$
\begin{aligned}
w_{d 2}(\delta) & =F_{d 2}(\delta)(-\delta) \\
& =-\frac{8}{3} \pi \gamma R\left(\left(\frac{\varepsilon}{\varepsilon-\delta}\right)^{2}-0.25\left(\frac{\varepsilon}{\varepsilon-\delta}\right)^{8}\right) \delta
\end{aligned}
$$

Combining equation (1) and (3), the total Lennard Jones potential energy in all the non contacting asperities can be obtained as

$$
\begin{aligned}
W_{d 2} & =N \int_{-\infty}^{d} w_{d 2}(\delta) \phi(z) d z \\
& =-N \int_{-\infty}^{d} \frac{8}{3} \pi \gamma R\left(\left(\frac{\varepsilon}{\varepsilon-\delta}\right)^{2}-0.25\left(\frac{\varepsilon}{\varepsilon-\delta}\right)^{8}\right) \delta \phi(z) d z
\end{aligned}
$$

Dividing both side by $\mathrm{A}_{\mathrm{n}} \mathrm{K} \sigma$

$$
\begin{aligned}
\mathrm{W}_{\mathrm{d} 2}^{*} & =-\frac{8}{3} \pi(\eta \mathrm{R} \sigma)\left(\frac{\gamma}{\mathrm{K} \sigma}\right) \int_{-\infty}^{0}\left(\left(\frac{\varepsilon_{0}}{\varepsilon_{0}-\Delta}\right)^{2}-0.25\left(\frac{\varepsilon_{0}}{\varepsilon_{0}-\Delta}\right)^{8}\right) \Delta \phi(\Delta) \mathrm{d} \Delta \\
& =-\frac{8}{3} \pi \mathrm{A}_{0} \mathrm{~B}_{0} \int_{-\infty}^{0}\left(\left(\frac{\varepsilon_{0}}{\varepsilon_{0}-\Delta}\right)^{2}-0.25\left(\frac{\varepsilon_{0}}{\varepsilon_{0}-\Delta}\right)^{8}\right) \Delta \frac{1}{\sqrt{2 \pi}} \exp \left[-\frac{(\mathrm{h}+\Delta)^{2}}{2}\right] \mathrm{d} \Delta
\end{aligned}
$$

Total deformation work done, $\mathrm{W}_{\mathrm{d}}^{*}=\mathrm{W}_{\mathrm{d} 1}^{*}+\mathrm{W}_{\mathrm{d} 2}^{*}$

\subsubsection{Restoration work done}

During adhesive elastic impact, velocity of two bodies reach to zero at particular mean separation and total kinetic energy stored as a form of strain energy of the asperities. After end of deformation period, the strain energy is released in the form of kinetic energy through the elastic recovery of asperities during restoration period. During elastic recovery of each asperity, some amount of energy would be lost due to over come the pull off force, $2 \pi R \gamma$. The loss of energy would be evaluated following way.

$W_{l}(\delta)=N \int_{d}^{\infty}\left(\int_{0}^{\delta} F_{\text {pulloff }} d \delta\right) \phi(z) d z$ 


$$
\begin{aligned}
& =N \int_{d}^{\infty}\left(\int_{0}^{\delta} 2 \pi R \gamma d \delta\right) \phi(z) d z \\
& =2 \pi N R \gamma \int_{d}^{\infty} \delta \phi(z) d z
\end{aligned}
$$

Dividing both side by $\mathrm{A}_{\mathrm{n}} \mathrm{K} \sigma$

$$
\begin{aligned}
\mathrm{W}_{1}^{*} & =2 \pi(\eta \mathrm{R} \sigma)\left(\frac{\gamma}{\mathrm{K} \sigma}\right)_{0}^{\infty} \Delta \phi(\mathrm{z}) \mathrm{dz} \\
& =2 \pi \mathrm{A}_{0} B_{0} \int_{0}^{\infty} \Delta \frac{1}{\sqrt{2 \pi}} \exp \left[-\frac{(h+\Delta)^{2}}{2}\right] d \Delta
\end{aligned}
$$

So, Restoration work done $=$ Deformation work done - Loss of work done i.e. $\mathrm{W}_{\mathrm{r}}^{*}=\mathrm{W}_{\mathrm{d}}^{*}-\mathrm{W}_{1}^{*}$

\section{- Coefficient of Restitution}

Coefficient of restitution $=$ Restoration work done $/$ Deformation work done i. e. $e=\frac{W_{r}^{*}}{W_{d}^{*}}$

\section{RESULTS AND DISCUSSION}

In the numerical simulation, three type combined head disk surfaces coated with ZDOL lubricant thickness, $8 \AA, 12 \AA$, and 20 A respectively are considered, and for respective HDI, mechanical and tribolgical parameters are taken as input datas from Suh and Polycarpou work [7] as given in the Table.1 below.

Table.1Input data

\begin{tabular}{|c|c|c|c|}
\hline Combined Head Disk Surfaces & $\begin{array}{c}\text { Smooth } \\
\text { HDI }\end{array}$ & $\begin{array}{c}\text { Intermidiate } \\
\text { HDI }\end{array}$ & $\begin{array}{c}\text { Supersmooth } \\
\text { HDI }\end{array}$ \\
\hline Asperity density $\eta\left(\mathrm{m}^{-2}\right)$ & $17.955 .10^{12}$ & $17.983 .10^{12}$ & $18.183 .10^{12}$ \\
\hline Asperity radius $\mathrm{R}(\mathrm{m})$ & $7.561 .10^{-6}$ & $6.712 .10^{-6}$ & $6.311 .10^{-6}$ \\
\hline $\begin{array}{c}\text { Stadandard deviation of asperity } \\
\text { height } \sigma(\mathrm{m})\end{array}$ & $0.246 .10^{-9}$ & $0.272 .10^{-9}$ & $0.291 .10^{-9}$ \\
\hline Surface energy $\gamma(\mathrm{N} / \mathrm{m})$ & 0.09 & 0.09 & 0.09 \\
\hline Modulus of elasticity $\mathrm{K}\left(\mathrm{N} / \mathrm{m}^{2}\right)$ & $148.79 .10^{9}$ & $148.79 .10^{9}$ & $148.79 .10^{9}$ \\
\hline Yield stress Y $\left(\mathrm{N} / \mathrm{m}^{2}\right)$ & $2.5 .10^{9}$ & $2.5 .10^{9}$ & $2.5 .10^{9}$ \\
\hline Equilibrium distance $\varepsilon(\mathrm{m})$ & $4.10^{-10}$ & $4.10^{-10}$ & $4.10^{-10}$ \\
\hline Lubricant thickness $\mathrm{t}(\mathrm{m})$ & $8.10^{-10}$ & $12.10^{-10}$ & $20.10^{-10}$ \\
\hline Roughness parameter $\mathrm{A}_{0}$ & $33.396 .10^{-3}$ & $32.831 .10^{-3}$ & $33.393 .10^{-3}$ \\
\hline Surface energy parameter $\mathrm{B}_{0}$ & $24.588 .10^{-4}$ & $22.238 .10^{-4}$ & $20.786 .10^{-4}$ \\
\hline Asperity radius parameter $\mathrm{R}_{0}$ & 30736 & 24676 & 21687 \\
\hline Equilibrium distance parameter $\varepsilon_{0}$ & 1.626 & 1.471 & 1.375 \\
\hline Lubricant thickness parameter $\mathrm{t}_{0}$ & 3.25 & 4.41 & 6.87 \\
\hline
\end{tabular}

In this study, adhesive impact of head disk is considered in the field of Lennard-Jones potential. Deformation work done is developed due to combination of elastic potential energy for contacting asperities and LJ potential energy for noncontacting asperities. Fig.3 displays variation of deformation work done verses mean separation for different combination of head-disk surfaces. From the Fig.3, its found that deformation work done increases with decrement of mean separation upto $h=0$ and thereafter deceases to a finite value. Generally, as mean separation deceases, more number of contacting asperities are deformed such a way elastic potential energy is stored gradually in the form of strain energy and more number of nocontacting asperities stores LJ potential energy. It also indicates that as smoothness of head-disk surfaces increases, the deformation work done decreases for a particular mean separation. Hence, rough head-disk surfaces are much more capable of storing deformation work done. Fig.4 shows variation of lost work done with mean separation in presence of adhesion. 
As head-disk surfaces very smooth, adhesion force is predominating at the interface during recording. Due to close proximity recording, if somehow, head touches the disk, immediately, it would be rebounded from the disk through dissipation of kinetic energy to overcome adhesion at interface. The figure indicates, lost work done increases with decrement of mean separation. During deformation period, as mean separation decreases, the number of contacting asperities increases at the real area of contact of head-disk surfaces, and during start of restoration period, more amount of kinetic energy would be lost to overcome adhesion at the real area of contact.

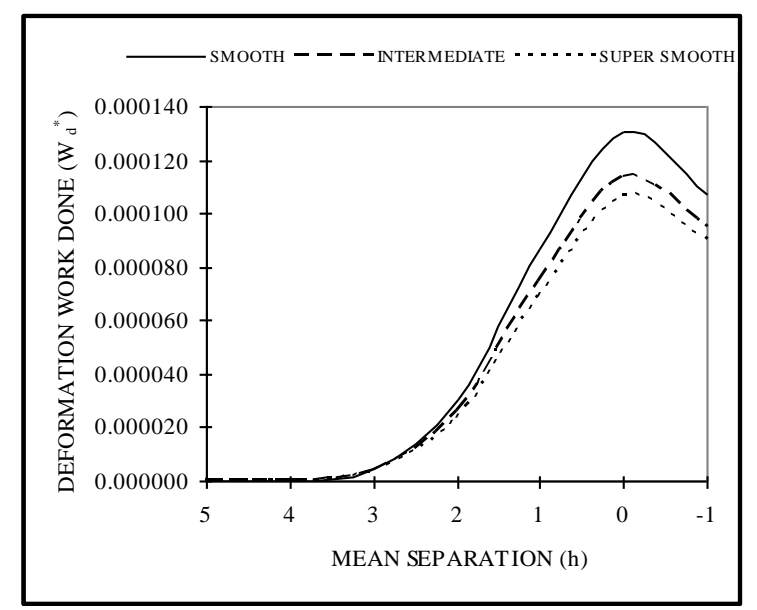

Fig.3 Deformation work done vs Mean separation

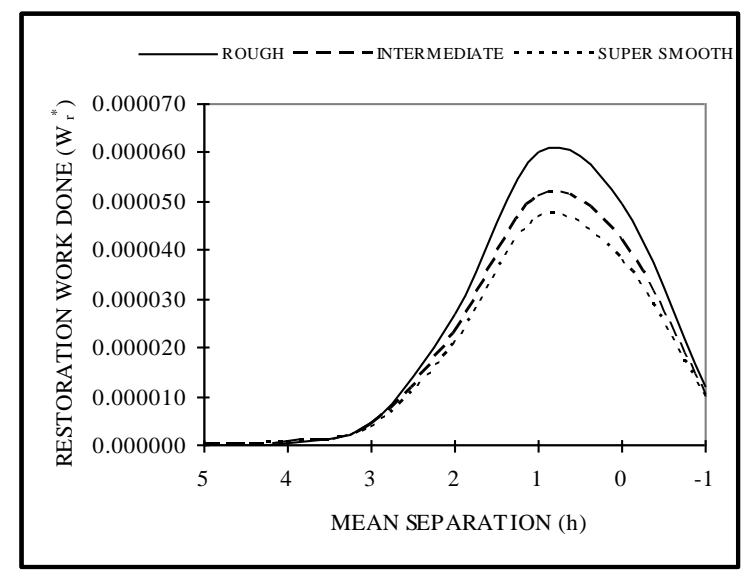

Fig.5 Restoration work done vs Mean separation

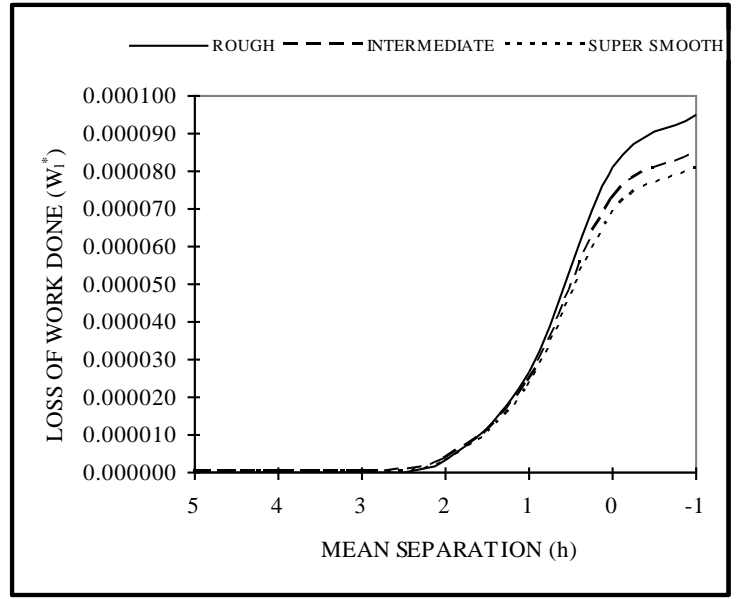

Fig.4 Loss of work done vs Mean separation

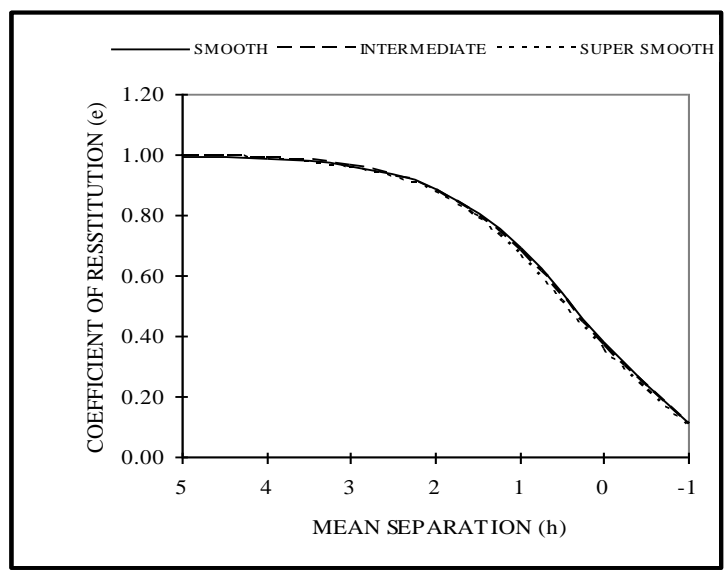

Fig.6 Coefficient of restitution vs Mean separation

Fig.5 depicts variation of restoration work done with mean separation for head-disk interfaces. Restoration work done is the algebraic sum of deformation work done and lost work done due to adhesion. From the variation of restoration work done, its found that it reaches to maximum value for $\mathrm{h}=0.5$ and sharply, decreases to almost zero value for $\mathrm{h}=-1$. So, it indicates during very nearer recording, if there is high impact, head and disk would stick to each other owing to high adhesion resulting restoration work done almost reaches to zero. The catastrophic phenomenon of head-disk seizure is more clearly found from Fig.6 which plots variation of coefficient of restitution with mean separation. The COR is gradually decreases with decrement of mean separation and it approaches to almost zero value. So, high adhesive impact for micromechanical surface contact like head-disk have almost similar tendency to complete plastic impact.

\section{CONCLUSION}

From the study of adhesive impact of head-disk surfaces, its found coefficient of restitution gradually decreases with decrement of mean separation and it approaches to near value of zero. So, it indicates possibility of seizure of head with disk is very high for very close impact and there are very less chance of separation during restoration period. Similar type of phenomenon occurs incase of plastic impact of soft body where coefficient of restitution is zero owing to stick each other after impact. Moreover, Graph for COR vs Separation represents unique characteristic of adhesive impact for any clean and smooth surface contact like head-disk surfaces. 


\section{REFERENCES}

[1] R R Katta, A A Polycarpou, J V Hanchi, and M Roy, Analytical and Experimental Elastic-Plastic Impact Analysis of a Magnetic Storage Head-Disk Interface, ASME Journal of Tribology, 2009, 131, 011902 1-10

[2] W R Chang and F F Ling, Normal impact model of rough surfaces, ASME Journal of Tribology, 1992 , $114,439-447$

[3] P Sahoo and S K Roy Chowdhury, Normal impact of rough surfaces in presence of adhesion, Tribology International, 2004, 37, 667-675

[4] B V Derjaguin, V M Muller, and YU P Toporov, Effect of contact deformations on the adhesion of particles, Journal of Colloid Interface Science, 1975, 53, 314-326

[5] V M Muller, B V Derjaguin and YU P Toporov, On two methods of calculation of the force of sticking of an elastic sphere to a rigid plane, Colloids and Surfaces, 1983, 7, 251-259

[6] J A Greenwood and J B P Williamson, Contact of nominally flat surfaces. Proc. R. Soc. Lond., A, 1966, 295, 300-319

[7] A Y Suh and A A Polycarpou, Effect of molecularly thin lubricant on roughness and adhesion of magnetic disks intended for extremely high-density recording, Tribology Letter, , 2003, 15, 365-376 